\section{Animal models of retinal detachment and reattachment: identifying cellular events that may affect visual recovery}

GP Lewis ${ }^{1}$, DG Charteris $^{3}$, CS Sethi ${ }^{3}$ and SK Fisher ${ }^{1,2}$

\begin{abstract}
Retinal detachment continues to be a significant cause of visual impairment, either through the direct effects of macular detachment or through secondary complications such as subretinal fibrosis or proliferative vitreoretinopathy. Animal models can provide us with an understanding of the cellular mechanisms at work that account for the retinopathy induced by detachment and for the generation of secondary effects. As we understand the mechanisms involved, animal models can also provide us with opportunities to test therapeutic agents that may reduce the damaging effects of detachment or improve the outcome of reattachment surgery. They may also reveal information of use to understanding other causes of blindness rooted in retinal defects or injuries. Understanding the effects of detachment (and reattachment) are likely to become even more important as surgeons gain skills in subretinal surgical techniques and macular translocation, both of which will generate short-lived detachments. Here we discuss the fundamental events that occur after detachment, present changes associated with reattachment, and discuss retinal changes that may affect the return of vision. Eye (2002) 16, 375-387. doi:10.1038/ sj.eye. 6700202
\end{abstract}

Keywords: retinal detachment; retinal reattachment; photoreceptors; retinal pigmented epithelium; synaptic plasticity; Müller cells

\section{Introduction}

Animal models provide us with the only means to study in a systematic and controlled way, the cellular responses of the retina to detachment and reattachment. Two major goals of using animal models for this research are to find those models that best mimic human detachments in terms of cellular responses, and to find those models that can best provide clues about the various biological mechanisms involved in the complex cellular responses. Then these models can be used to discover ways of manipulating the retinal environment to improve visual outcome or reduce the likelihood of complications after reattachment surgery. The retinas of different species react differently to detachment. Thus, the selection of species to use in any one study must be done carefully. Comparative studies in species that react differently to humans are valuable because they may provide us with important information about cellular mechanisms underlying the complex responses to detachment and reattachment. However, there also is a real need for the study of human tissue in parallel with the study of animal models. The study of human tissue is often complicated by a lack of precise information on the duration or cause of the detachment, by the presence of multiple clinical procedures, or by secondary manifestations such as proliferative vitreoretinopathy (PVR). Nonetheless, this tissue is highly valuable because even with limitations it will help identify those animal models that do accurately predict results in the human eye, and suggest hypotheses to test in the experimental models.

Although we have studied detachment in primates, rabbits, and ground squirrels, the
${ }^{1}$ Neuroscience Research Institute

University of California Santa Barbara CA, USA

${ }^{2}$ Molecular, Cellular \& Developmental Biology University of California Santa Barbara CA, USA

${ }^{3}$ Vitreoretinal Research Unit Moorfields Eye Hospital City Road, London, UK

Correspondence: SK Fisher, PhD

Neuroscience Research Institute

University of California Santa Barbara CA 93106, USA Tel: (805) 8933611 Fax: (805) 8932005

E-mail: fisher@ lifesci.ucsb.edu 
feline retina remains the major focus of our experimental work. The feline retina, like the human retina outside of the fovea, is rod-dominated. ${ }^{1}$ It is a retina extensively studied by both anatomists and physiologists. Like the human retina, it has a robust intraretinal circulation that is excluded from the photoreceptor layer (which is served by the choroidal cirulation). The eye of the domestic cat is relatively large, allowing for easy surgical access, and this in turn, allows for the production of a limited detachment in one specific region or a total detachment. Its reflective tapetum improves retinal observation during surgical procedures. Our procedures for producing retinal detachment ${ }^{2}$ involve removing the lens and vitreous and then slowly infusing fluid into the interphotoreceptor space through a glass micropipette drawn to a tip diameter of about $100 \mu \mathrm{m}$. It could be argued that this does not model a rhegmatogenous detachment because the relatively small hole left by the pipette is the only retinal 'break.' However, the limited numbers of studies done on human detachment or reattachment tissue all validate this as a model producing the same cellular responses as rhegmatogenous detachment. Thus, in the description below we simply use the term, 'detachment,' to describe our procedures.

The rabbit retina has about the same rod/cone population as that of the feline retina, however its retinal circulation is very different since it has no intraretinal vessels and the inner retina is perfused by vasculature that lies on its vitreal surface. The rabbit retina tends to degenerate very rapidly and almost completely after detachment. ${ }^{3}$ Detachments can be made in the eyes of common laboratory rodents, but the small size of the eye makes placement and control of the detachment difficult, and reattachment surgery even more difficult. ${ }^{4}$ Most importantly the retina of rodents does not seem to mount a vigorous Müller cell response to detachment. Indeed, the Müller cells of the cone-dominant ground squirrel show almost none of the traditional signs of glial reactivity after detachment. The retinas of primates with a fovea are probably ideal models for human detachments. ${ }^{5,6}$ but there are obvious cost disadvantages, as well as health considerations and ethical concerns that influence the use of these species.

All of the original research presented below was approved by the Animal Care Council of the University of California, Santa Barbara, and in accordance with the guidelines provided by the ARVO Statement for the Use of Animals in Ophthalmic and Vision Research.

\section{Results and Discussion}

Retinal detachment (except in a few species such as the rabbit) does not produce a retinal degeneration per se. The cellular 'degeneration' that ensues is limited to photoreceptors and this usually does not result in complete destruction of these cells, but rather a process that we have termed 'deconstruction' of all, and the death of some. ${ }^{7}$ We have preferred the term 'retinopathy,' to describe the results of detachment because it involves a variety of complex but characteristic cellular responses in many different retinal cell types. ${ }^{8}$ Most importantly, many of the events initiated by detachment can be halted or reversed by reattachment, or by providing certain specific biological factors such as the trophic factor known as 'brain-derived neurotrophic factor' (BDNF), or an increase in environmental oxygen (hyperoxia). ${ }^{\text {? }}$

\section{The photoreceptor/RPE interface}

The photoreceptor/retinal pigment epithelial (RPE) interface has received the greatest attention in studies of retinal detachment and reattachment. The rationale being, of course that the photoreceptor outer segments are the source of visual transduction ultimately resulting in 'seeing.' Very early it was demonstrated that outer segments would degenerate when detached from the RPE, and re-grow when the retina was reattached..$^{5,10}$ The early studies also de-emphasized the imperfections and variability in the results of regeneration after reattachment, emphasizing instead the ability of outer segments to regenerate, an important observation that explained the return of vision after reattachment.

The RPE/photoreceptor interface after long-term reattachment has been described as having a 'patchwork' appearance. ${ }^{11}$ This refers to the fact that even after relatively short periods of detachment the extent of outer segment regrowth varies over long stretches of the reattachment, as does outer segment alignment, and that the subretinal space has frequent islands of RPE proliferation, Müller cell processes, and collections of unidentified cells. Also, the RPE apical surface does not return to a completely normal morphology, particularly where it contacts cones with a complex of ensheathing processes. The fact that recovery across the interface is not uniform is easily illustrated in the feline retina by using specific molecular probes to cones and the RPE (Figure 1). In these experiments the retina was detached for either 1 $\mathrm{h}$ or 1 day and then the animals killed on day 3 , or detached for 3 days and then killed on day 28. In the examples shown in Figure 1, the RPE is labeled with 

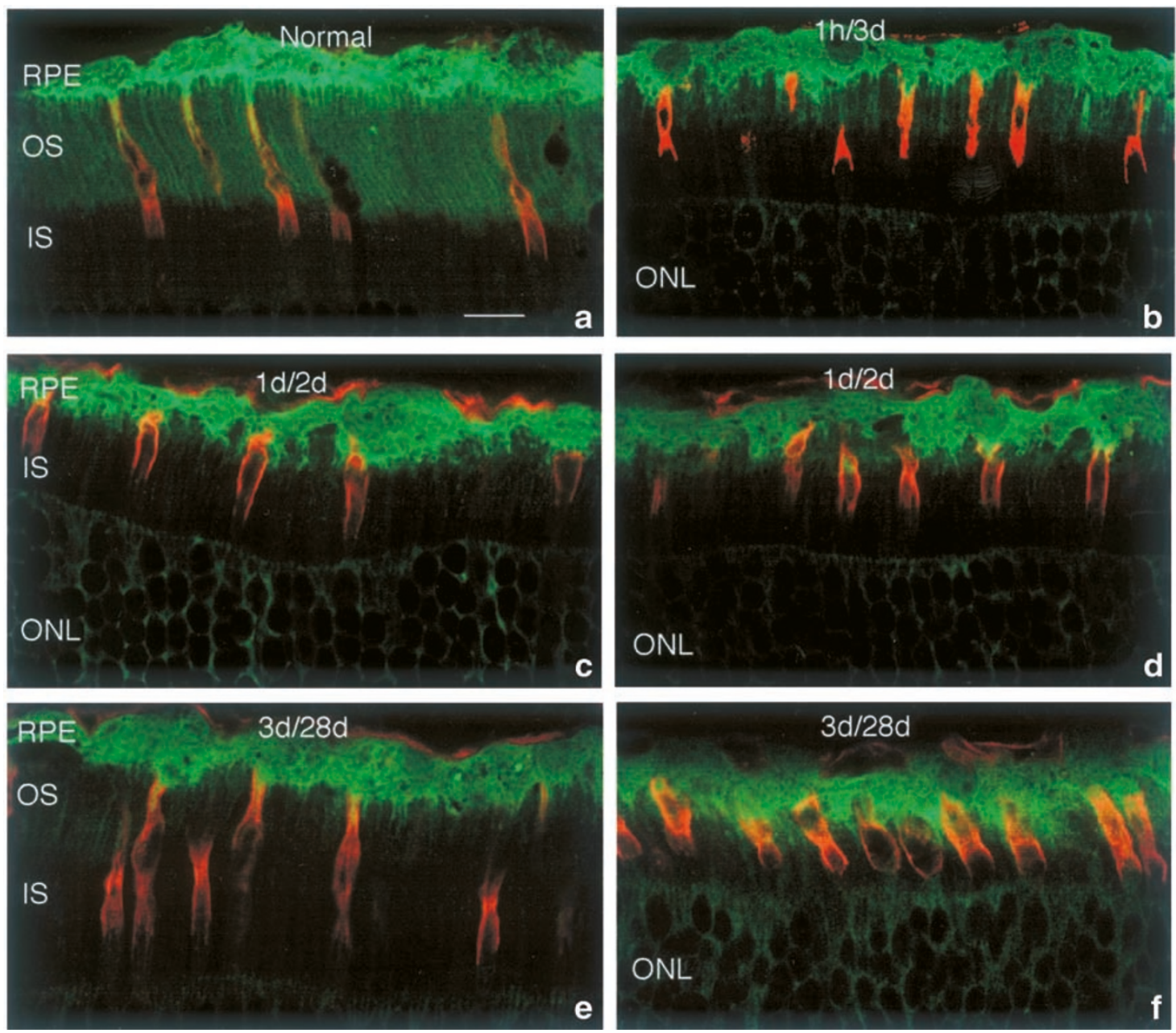

Figure 1 Confocal microscope images of retinas immunolabeled with anti-cellular retinaldehyde binding protein (CRALBP; green) and biotinylated-peanut agglutinin (PNA; red). (a) Normal retina. Anti-CRALBP labels the retinal pigment epithelium (RPE) and the long apical microvilli that extend down over the cone outer segments forming the cone sheath; PNA labels the specialized cone matrix around the cone outer segments. (b) Retina detached for $1 \mathrm{~h}$ and reattached for 3 days (1h/3d). (c, d) Retinas detached for 1 day and reattached for 2 days $(1 \mathrm{~d} / 2 \mathrm{~d})$. In (b), (c), and (d), the apical microvilli are greatly truncated or lost and the cone sheaths are shorter and less delicate; the cone matrix domain appears shorter and as if it merely abuts the RPE apical surface. (e, f) Retinas detached for 3 days and reattached for 28 days ( $3 \mathrm{~d} / 28 \mathrm{~d}$ ). Some regions look relatively normal except for the absence of the highly organized RPE processes (e). Other regions continue to show shortened outer segments with an abnormal relationship to the RPE (f). OS, outer segments; IS, inner segments; ONL, outer nuclear layer. Scale bar, $10 \mu \mathrm{m}$.

an antibody to cellular retinaldehyde binding protein (CRALBP, a gift from Dr John Saari, Seattle, WA, USA), and the cones with the lectin peanut agglutinin (PNA, Vector Labs, Burlingame, CA, USA) which specifically labels the matrix surrounding cone outer segments. Visualization is by fluorescence imaging and optical sectioning of $100 \mu \mathrm{m}$ thick sections with the laser scanning confocal microscope. Because the antibody concentrations and microscope settings are kept constant, the observed fluorescence can be interpreted in a semi-quantitative way. First of all, the intensity of labeling for anti-CRALBP in the RPE decreases if the retina is detached for even short periods of time. The labeling intensity in the short-term detachments and reattachments (Figure 1a-d) is equivalent to that seen in detached retina, and it remains depressed even in the relatively long-term 28 day reattachments (Figure 1e, f). In the feline retina cones enjoy a highly specialized anatomical interaction with the RPE. This is evident in the very fine antiCRALBP labeled processes that extend down over the cone outer segment in the normal retina (Figure 1a). In short-term reattachments these specialized processes are greatly truncated or missing altogether (Figure $1 \mathrm{~b}-$ d); in some areas of long-term detachment they seem to re-form, although rarely with the same degree as in the normal eye (Figure 1e), while in other areas they do not re-form at all (Figure 1f). Concomitant with the 
rapid change in the RPE apical processes, cone outer segments degenerate rapidly, and remain much shorter than normal in the short-term reattachments. In some areas of long-term reattachments cones look relatively normal (Figure 1e), while in other areas they are greatly shortened (Figure 1f). This variability in recovery of cones is common and may account for some of the variability observed in visual recovery observed in human macular detachments.

\section{Outer segment recovery}

Outer segments may take a very long time to recover their pre-detachment length. In the experiments described above, the width of the outer segment zone was measured. Because the cat retina is rod dominated and rods are significantly longer than cones, this measurement reflects the length of the rod outer segments. The results are shown in Figure 2. The regrowth of the rods is obvious from these data.

However, even at a month of reattachment these have not recovered their pre-detachment length. In reattachment experiments with rhesus macaques, Guerín and colleagues ${ }^{12}$ reported that both rods and cones had recovered less than $50 \%$ of their length after 30 days of reattachment. Only at 150 days of reattachment were lengths statistically the same as in the normal animals. Even at this time the distribution of outer segment lengths was different from normal, and many of the cones were only $12 \mu \mathrm{m}$ long, or about $2 / 3$ of their length in the controls.

Outer segment lengths

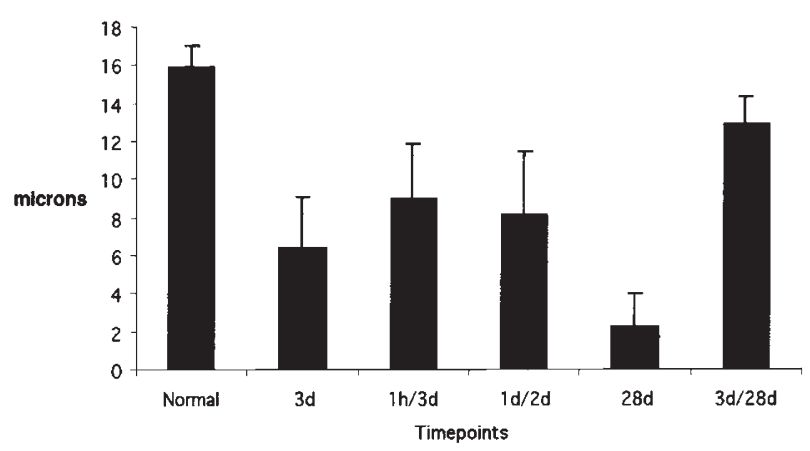

Figure 2 Graph of outer segment length measurements. Considerable outer segment shortening occurs at day 3 following a short-term detachment interval of $1 \mathrm{~h}(1 \mathrm{~h} / 3 \mathrm{~d})$ or 1 day $(1 \mathrm{~d} / 2 \mathrm{~d})$. Retinas that were detached for 3 days and reattached for 28 days (3d/28d) have outer segment lengths that are considerably longer than the 28 day (28d) detachments without reattachment, although still slightly shorter than normal outer segments.
TUNEL labeling

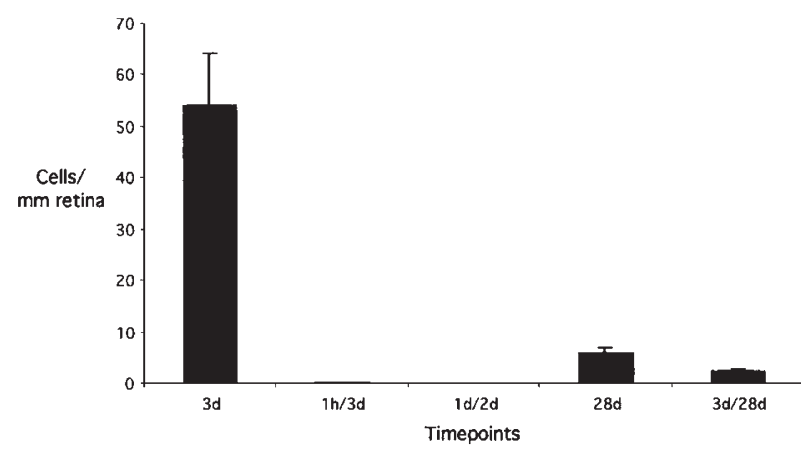

Figure 3 Graph of the number of TUNEL labeled cells counted per millimeter of retina. TUNEL labeling is essentially abolished when retinas are reattached after either a $1 \mathrm{~h}(1 \mathrm{~h} / 3 \mathrm{~d})$ or 1 day $(1 \mathrm{~d} / 2 \mathrm{~d})$ detachment and examined on day 3 , compared to TUNEL labeling at 3 days after detachment (3d) without reattachment. TUNEL labeled cells are still observed after 28 days of reattachment in retinas that were detached for 3 days $(3 \mathrm{~d} / 28 \mathrm{~d}$ ) although slightly less than observed in 28 day detachments (28d) without reattachment.

\section{Photoreceptor cell death}

Photoreceptor cell death has been shown to occur by apoptosis after detachment in both the feline ${ }^{13}$ and human retina, ${ }^{14}$ although this does not rule out their death by other means. There is a steady decline in the thickness of the outer nuclear layer (ONL) if the retina remains detached. ${ }^{15,16}$ Although the actual peak of TUNEL positive cells appears to occur at around day 3 of detachment, ${ }^{13}$ they remain in the retina even at a month after the detachment event, indicating that cell death is still occurring.

Reattachment has a profound effect on cell death.

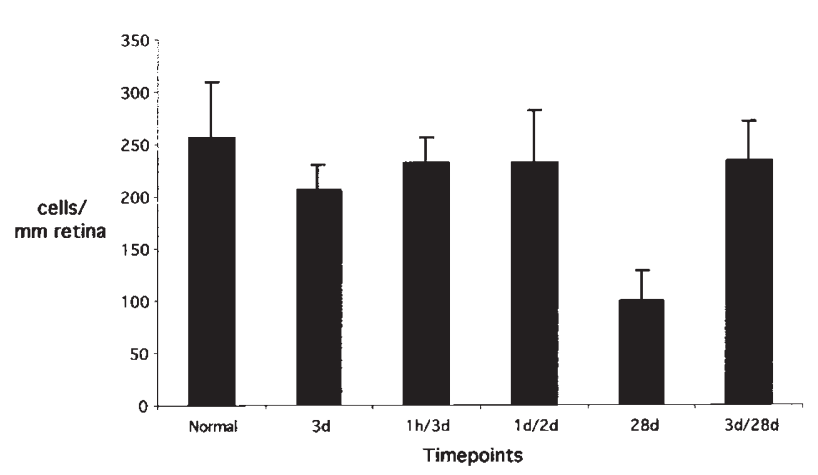

Figure 4 Graph of the number of photoreceptor nuclei counted in the outer nuclear layer per millimeter of retina. Only a slight decrease in the number of cells is observed in both the short and long-term detachments followed by reattachment $(1 \mathrm{~h} / 3 \mathrm{~d}$; $1 \mathrm{~d} / 2 \mathrm{~d} ; 3 \mathrm{~d} / 28 \mathrm{~d}$ ) whereas there is a significant reduction in the number of cells in 28 day (28d) detachments without reattachment. 

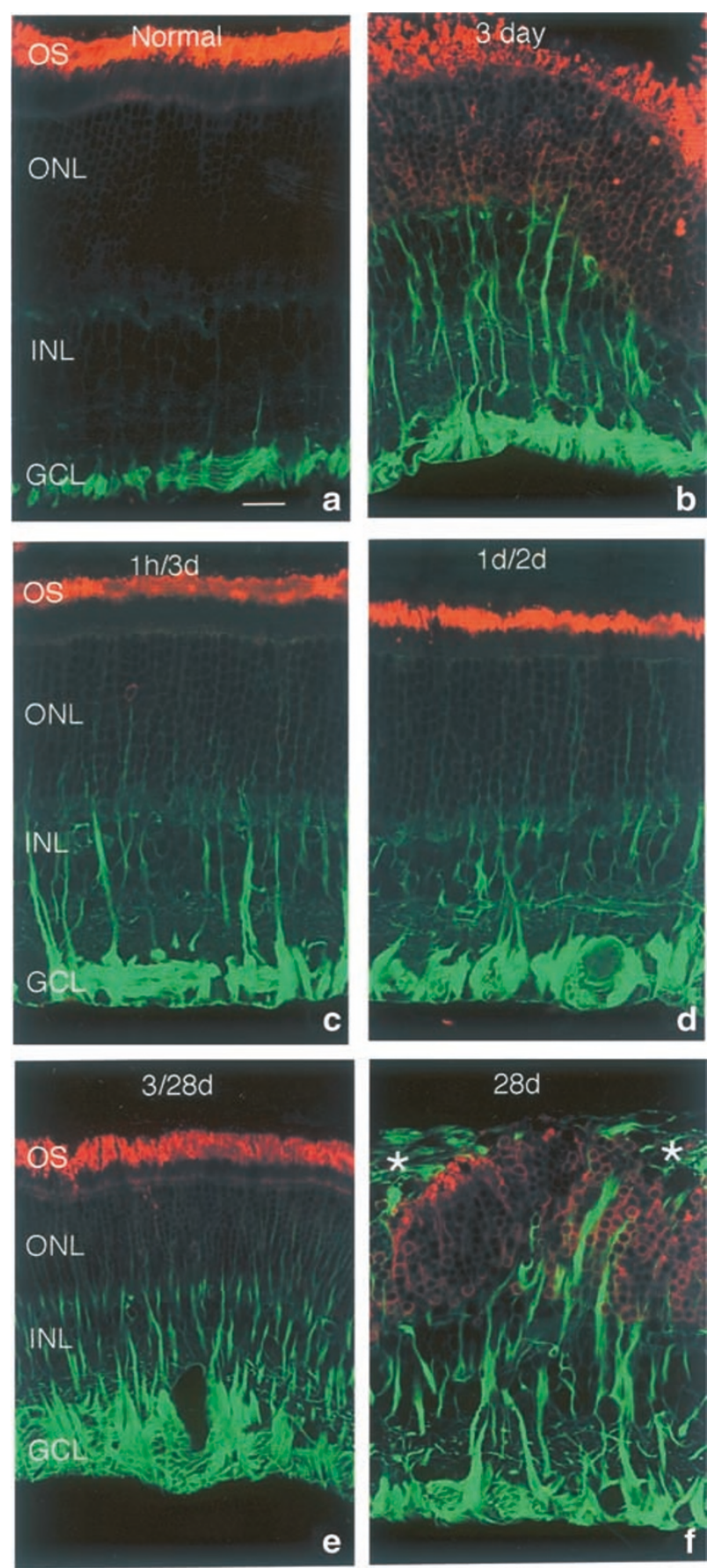

Figure 5 Confocal images of retinas immunolabeled with antibodies to rod opsin (red), and glial fibrillary acidic protein (GFAP; green). (a) Normal retina. Anti-rod opsin labeling is restricted to the outer segments (OS); anti-GFAP labels intermediate filaments in Müller cell endfeet and in the processes of astrocytes. (b) 3-day detached retina. Anti-rod opsin labels the truncated OS and the rod cell bodies in the outer nuclear layer (ONL); anti-GFAP labels Müller cells extending into the ONL. (c, d) Retinas detached for $1 \mathrm{~h}$ or 1 day and reattached for 3 days or 2 days, respectively ( $1 \mathrm{~h} / 3 \mathrm{~d} ; 1 \mathrm{~d} / 2 \mathrm{~d})$. Anti-rod opsin labels the shortened OS with very little labeling present in the ONL; antiGFAP labeling in the Müller cells extends into the ONL. (e) Ret-
Essentially no TUNEL-positive cells were observed in the reattached retinas with the short-term detachments (Figure 3), while in the retinas detached for 3 days there are still TUNEL-positive cells in the ONL at 28 days of reattachment. The number of cell bodies in the ONL gives an overview of the effects of cell death, and here the profound effect of reattachment is clearly shown (Figure 4). The ability of cones to survive detachment is a critical issue for patients with foveal detachments. Determining whether rods and cones have a differential capacity for survival in longer term detachments is a serious issue, and a difficult one to study because cones appear to rapidly lose the expression of various molecules that are used currently as specific markers. ${ }^{17,18}$ Cones may even have specialized mechanisms that allow them to survive detachment but, in fact, very little is known about these important cells. Ideally cone survival should be studied in the fovea of primate species, but as indicated earlier this is problematic. The conedominated retinas of the ground squirrel may form a reasonable alternative to primates as a model system for the responses of cones. We have recently characterized the cellular and ERG ${ }^{19}$ responses of the retina in this species to detachment, and have documented the ability of hyperoxia to rescue its cone photoreceptors. ${ }^{20}$ While the responses of the squirrel cones to detachment are similar to those in the cat retina, the Müller cells behave in a very different way to those in cat (Linberg et al, in press; Sakai et al, in press). This raises the question as to whether the differences are specifically related to the high density of cones in this retina (and thus reflective of the reactions of Müller cells in human macula), or whether they simply reflect different responses in different species.

\section{Rod opsin redistribution}

In addition to outer segment degeneration, the deconstruction of the rods involves a number of other characteristic responses to detachment. Only two will be detailed here because we use both to measure the effectiveness of proposed treatment to mitigate the effects of detachment. The first response is the

ina detached for 3 days and reattached for 28 days (3/28). Antirod opsin labels the almost normal appearing outer segments; anti-GFAP labeling in Müller cells extends into the ONL. (f) Retina detached for 28 days (28d). Anti-rod opsin labels OS fragments as well as inner segments (IS) and rod cell bodies in the ONL; anti-GFAP labeling in the Müller cells extends through the entire width of the retina and into Müller cell processes forming a scar $\left(^{*}\right)$ in the subretinal space. INL, inner nuclear layer GCL, ganglion cell layer. Scale bar, $20 \mu \mathrm{m}$. 
'redistribution' of rod opsin molecules into plasma membrane that surrounds the inner portions of the rod photoreceptor cell. Immunolabeling with antibodies to rod opsin (a gift from Dr R Molday, Vancouver, BC, Canada) normally labels only the outer segment and the Golgi/RER in the inner segment (Figure 5a). As the OS begin to degenerate after detachment this labeling begins to increase in the plasma membrane around the inner segment, cell body, and synaptic terminal (Figure $5 b)$. This results in a bright signal from the ONL in retinas detached for more than a day, with the signal increasing in extent and intensity as detachment time increases. ${ }^{21}$ Reattachment can stop this process; treatment with BDNF or hyperoxia can diminish it. ${ }^{3,8}$ When the retina is reattached after $1 \mathrm{~h}$ of detachment there is almost no redistribution (Figure 5c). If it remained detached for a day before reattachment there is a significant response in some cells but not others (Figure 5d), although the response was greatly attenuated compared to that in the retina that remained detached for 3 days (Figure 5b). Although the mechanisms of this response are unknown, we can speculate that detachment results in the cell being unable to normally assemble the components of an outer segment, so that newly synthesized opsin is directed into the plasma membrane instead of the disc membranes. This would mean that in the short-term experiments reattachment allows normal disc assembly to begin again very quickly. Alternatively if the opsin redistribution is not related to failed disc assembly, then its mechanism of production must be stopped by reattachment. Reattachment experiments in monkey suggest that rod disc assembly rates remain significantly depressed for at least 2 weeks and slightly depressed even at 30 days. It appears however, that the process of opsin redistribution is reversible. At 3 days of detachment there is significant immunolabeling for rod opsin throughout the ONL (Figure 5b). When the retina is reattached at this 3-day point and then allowed to recover until day 28, there is significant recovery of the rod outer segments and also a large decrease in the amount of opsin redistribution (Figure 5e). This is even more apparent when the labeling in Figure $5 \mathrm{e}$ is compared to that in $5 \mathrm{f}$ where the retina remained detached for 28 days. The possibility that rod opsin is 'stored' in the plasma membrane and then made available for disc production once the retina is reattached is intriguing.

\section{Cone opsin redistribution}

Cones react similarly, but not identically to rods. They show opsin redistribution very quickly, within a day of detachment, but this redistribution is usually not as extensive as that of rod opsin (Figure 6a, b). Labeling (antibodies to cone opsins were gifts from Dr J Nathans, Baltimore, MD, USA) generally extends only around the cell body, occasionally outlining the synaptic terminal. As in the rods, the redistribution is prevented by reattachment (Figure 6c, d, e). A major difference between rods and cones emerges in detachments of 7 days or longer. In this case rod opsin immunolabeling would be extensive in surviving outer segments, in the plasma membrane of the IS, ONL, and synaptic layer (Figure 5f), while at this time there is virtually no labeling detected for cone opsins (Figure 6f). If rods store opsin in the plasma membrane, then use this in disc production in the reattached retina, the labeling data would suggest that cones have a different mode of operation after detachment. Either cone opsin production stops, or if it continues then it may be destroyed before it has a chance to be inserted into the plasma membrane.

\section{Photoreceptor synaptic terminals}

Rods One of the most dramatic responses to detachment is the retraction of the rod synaptic terminals. This was first reported by ultrastructural analysis in $1983,{ }^{15}$ but its extent was difficult to appreciate with the limiting sampling that occurs in the ultrathin sections used by this technique. Immunostaining with antibodies to synaptic proteins, however, provides a picture of just how extensive the response can be, and also how quickly it can occur. ${ }^{22}$ In the earlier study it was thought that this was a response to long-term detachments, when in fact it is recognizable in a retina detached for a single day. The response is illustrated in Figure 7, where the synaptic terminals are labeled with an antibody to synaptophysin (DAKO, Carpinteria, CA, USA), a synaptic vesicle protein. In the normal retina these form a densely packed, orderly band along the photoreceptor border of the outer plexiform layer (OPL, Figure 7a). In a 3 day detachment this labeling is now interrupted and bright labeling occurs high in the ONL (Figure 7b). By electron microscopy we know that the labeling in the ONL represents almost entirely rod terminals that appear to have 'retracted,' back towards the cell body. ${ }^{22}$ Their synaptic nature is recognizable by EM, but their synaptic organization has changed substantially from the normal complex arrangement within the rod spherule invagination. ${ }^{15,22}$ This pattern persists, becoming even more widespread in a 28 day detachment (Figure 7f). If the retina is reattached within a day, this terminal retraction does not occur (Figure 7c, d). If the retina is reattached at 3 days and examined at 1 month, we still find significant synaptic 

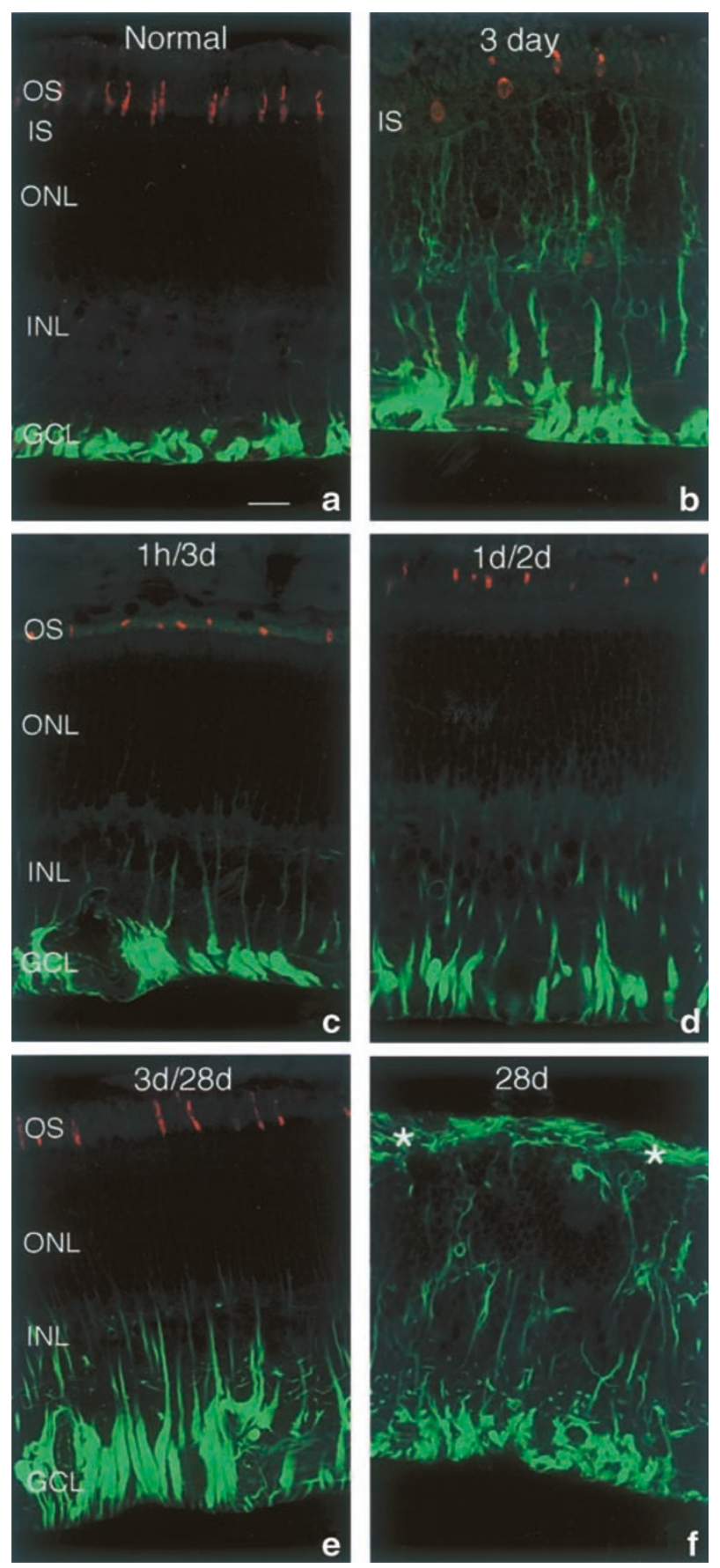

Figure 6 Confocal images of retinas immunolabeled with antibodies to $\mathrm{M} / \mathrm{L}$ cone opsin (red), and vimentin (green). (a) Normal retina. Anti-cone opsin labels the $\mathrm{M} / \mathrm{L}$ cones; anti-vimentin labels Müller cell endfeet. (b) 3 day detached retina. Anti-cone opsin labels M/L cone outer segment (OS) fragments and inner segments (IS); anti-vimentin labels Müller cells extending into the outer nuclear layer (ONL). (c, d) Retinas detached for $1 \mathrm{~h}$ or 1 day and reattached for 3 days or 2 days, respectively $(1 \mathrm{~h} / 3 \mathrm{~d} ; 1 \mathrm{~d} / 2 \mathrm{~d})$. Anti-cone opsin labels the shortened cone OS; anti-vimentin labels Müller cells extending into the inner nuclear layer (INL). (e) Retina detached for 3 days and reattached for 28 days (3/28). Anti-cone opsin labels the almost normal appearing cone OS; anti-vimentin labels Müller cells terminal labeling in the OPL. A comparison of Figures $7 \mathrm{~b}$ and $7 \mathrm{e}$ suggests that a remarkable recovery occurs in the reattached retina from its state at 3 days of detachment. Based on the short-term detachments (Figure $7 \mathrm{c}, \mathrm{d}$ ), reattachment presumably prevents the process of retraction. However, the organization of the layer of terminals in Figure $7 \mathrm{~b}$, and the occurrence of label in the ONL along with areas of a reattached retina that still appear somewhat 'abnormal' (Figure $7 \mathrm{e})$ suggest that recovery is not complete. The recovery of terminals in the reattached retinas also suggests that there is a re-growth of rod axons into the OPL and reformation of synapses. If so, this is a fairly astounding accomplishment for a portion of the adult CNS where growth and reformation of connections is presumed to be extremely limited. There is the possibility, however, that some of the loss and re-appearance of labeling in the OPL is not due to terminal retraction and regrowth, but due to disappearance of the synaptophysin protein and then its reappearance after reattachment. In some of these areas we see extension of rod axons into the inner retina, an event that does not occur in detached retinas. Thus, at least some rod axons appear to have the capability to grow. Some may re-extend to the OPL, while others grow past their target layer into the inner zones of the retina.

Cones It is difficult to pick out the large, but sparse cone pedicles among the dense array of rod spherules when the retina is immunolabeled with the antibody to synaptophysin. In the early morphological studies, it was our impression that cones did not withdraw their axon after detachment, but that the cone pedicles changed their shape, losing some of the complex synaptic invaginations that populate their base and assuming a somewhat 'flattened' appearance. ${ }^{15}$ As shown in Figure 8a, we can label cones from their outer segment to synaptic pedicles with an antibody to phosphodiesterase $\gamma$ (PDEy; gift from Dr B Fung, Los Angeles, CA, USA). The intensity of labeling with this antibody has already decreased at 3 days of detachment (Figure 8b), but still labels the cones sufficiently to demonstrate the beginning changes in shape of the cone synaptic terminals. In detachments of 7 days or longer, most of this labeling disappears. In the long-term reattachments, cones again label with the antibody, here clearly showing the variations in shape of the synaptic pedicles that can occur (Figure 8b, c, d).

extending into the inner portion of the ONL. (f) 28 day detached retina. No cone OS labeling is present; anti-vimentin labeling in Müller cells extends through the entire width of the retina and into a scar $\left(^{*}\right)$ in the subretinal space. GCL, ganglion cell layer. Scale bar, $20 \mu \mathrm{m}$. 

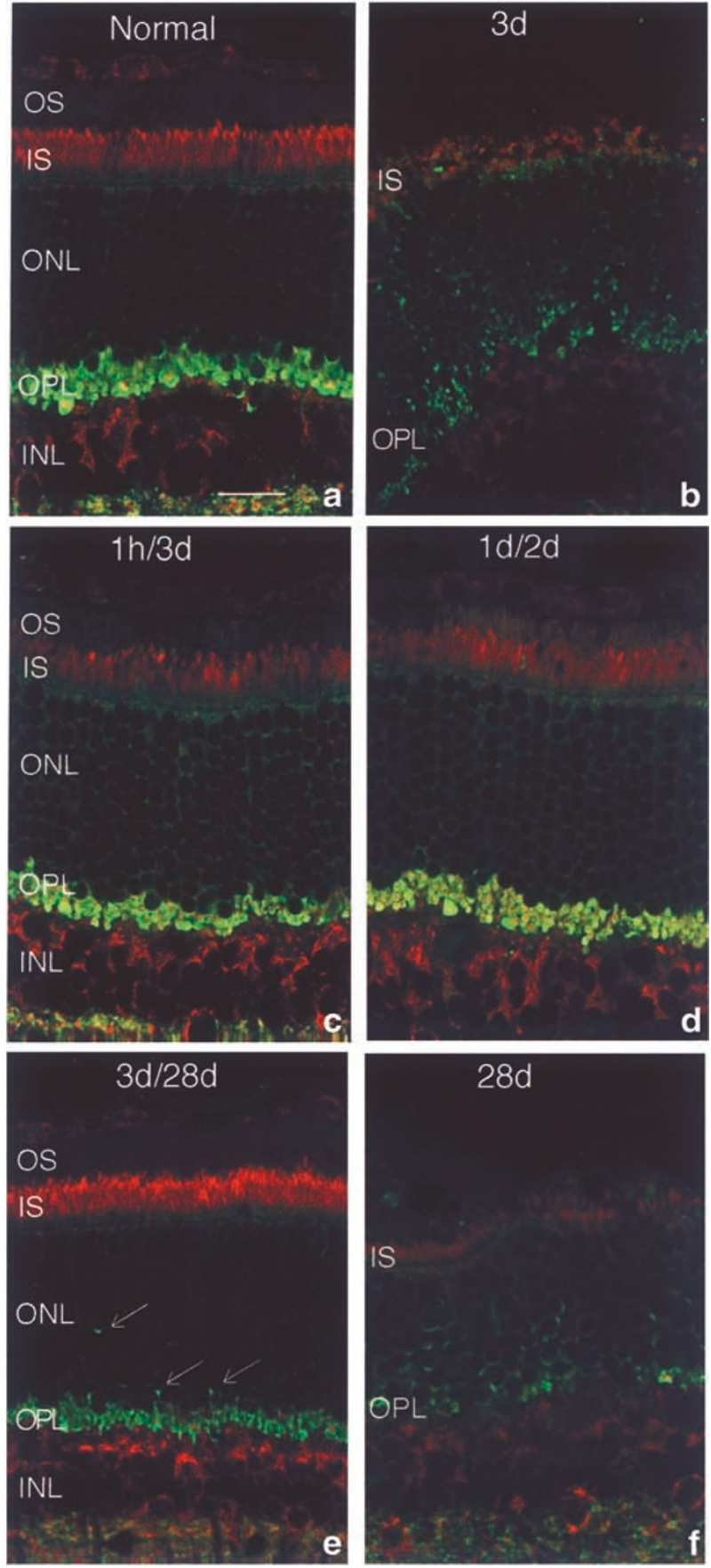

Figure 7 Confocal images of retinas immunolabeled with antibodies to cyotochrome oxidase (CO; red), and synaptophysin (green). (a) Normal retina. Anti-CO labels mitochondria in photoreceptor inner segments (IS) and cell bodies in the inner nuclear layer (INL); anti-synaptophysin labels synaptic vesicles in the terminals of rods and cones in the outer plexiform layer (OPL). (b) 3 day detached retina. Anti-CO labeling is diminished in the IS and INL; anti-synaptophysin labels rod terminals present in the outer nuclear layer (ONL) and fewer rod and cone terminals in the highly disrupted OPL. (c, d) Retinas detached for $1 \mathrm{~h}$ or 1 day and reattached for 3 days or 2 days, respectively $(1 \mathrm{~h} / 3 \mathrm{~d} ; 1 \mathrm{~d} / 2 \mathrm{~d})$. The labeling intensity of CO in the IS and INL is more comparable to normal; the distribution of anti-synap-
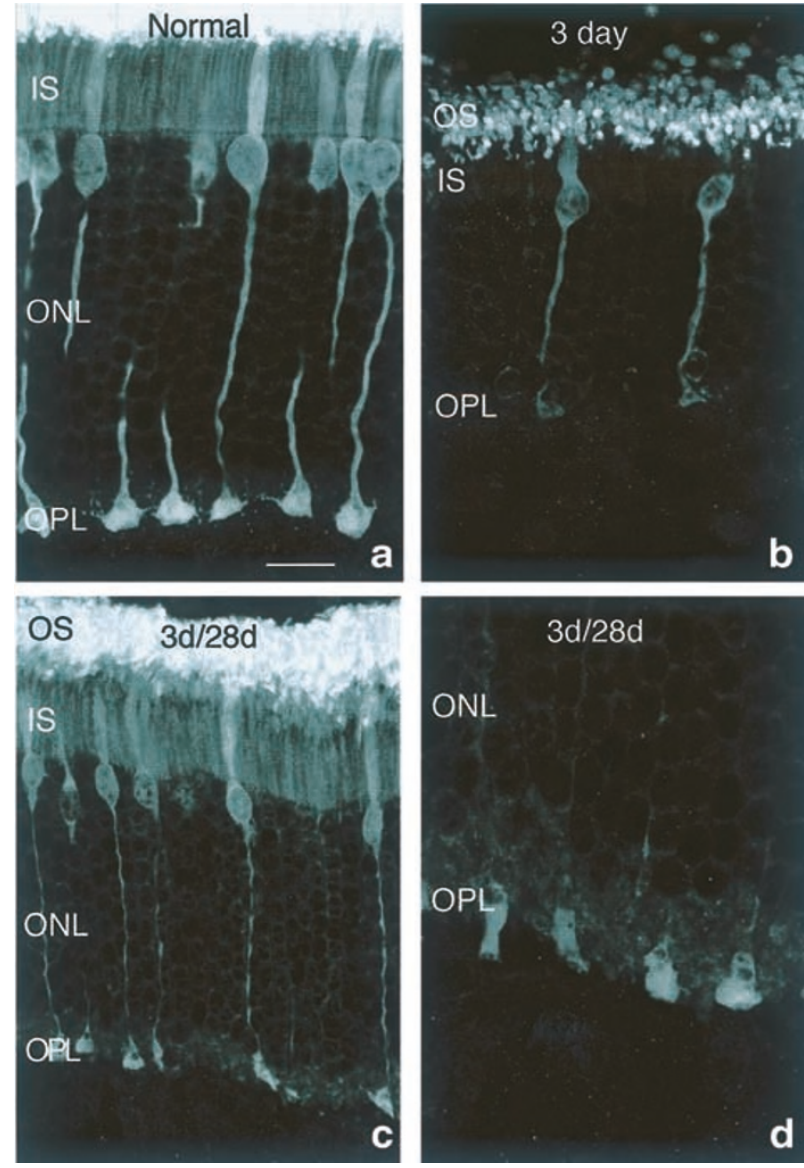

Figure 8 Confocal images of retinas immunolabeled with an antibody to phosphodiesterase gamma (PDE $\gamma$ ). (a) Normal retina. Anti-PDE $\gamma$ labels rod and cone outer segments (OS) as well as the entire cone cell. (b) 3 day detached retina. Anti-PDE $\gamma$ labeling illustrates the change in cone terminal shape as well as a decrease in labeling intensity of the cones. (c, d) Retinas detached for 3 days and reattached for 28 days ( $3 d / 28 d$ ). AntiPDE $\gamma$ labeling demonstrates the continued alteration in the shape of the cone terminals after long-term reattachment; some terminals appear flattened while others appear more elongated. IS, inner segments; ONL, outer nuclear layer. Scale bar, $10 \mu \mathrm{m}$.

Examination of the cone terminals by electron microscopy revealed that their synapses frequently appeared 'abnormal,' with a loss of the deep synaptic invaginations, and contacts occurring more on the terminal base than within the invaginations. ${ }^{15}$ Whether

tophysin labeling is also more like normal showing an organized OPL. (e) Retina detached for 3 days and reattached for 28 days (3/28). Anti-CO and synaptophysin labeling appear almost normal except for the few synaptophysin labeled terminals (arrows) present in the ONL (f). 28 day detached retina. The labeling intensity of anti-CO is very low; anti-synaptophsin labeling is present in retracted rod terminals in the ONL and in many fewer rod and cone terminals in the highly disrupted OPL. Scale bar, $20 \mu \mathrm{m}$. 
or how this change in the cone pedicles affects information flow through the retinal circuitry is unknown as is whether or not the synapses recover their normal morphology after long-term reattachment.

\section{Structural plasticity of second-order neurons}

The retina, as part of the CNS, is not a tissue associated with a great deal of structural plasticity. What we have discovered in the animal models (and now confirmed in human tissue) is that second order neurons, those that connect to the photoreceptors and either send information laterally (horizontal cells) or vertically (bipolar cells) through the retina, significantly remodel their processes in response to detachment. ${ }^{22}$ Presumably this remodeling occurs in response to the loss and retraction of photoreceptor terminals (in the case of rods). An antibody to protein kinase C (PKC; Amersham, Arlington Heights, IL, USA) is a specific marker for rod bipolar cells in several species. When normal feline retina is double-labeled with the antibody to synaptophysin and the antibody to PKC, it is possible to visualize the fine dendritic processes of the rod bipolar cells that terminate within the invaginations of the rod spherules (Figure 9a). When the retina is detached, and rod terminals retract toward the rod cell body, long fine processes extend from the rod bipolars into the ONL (Figure 9b, arrows). Often these can be traced to a retracted rod terminal (arrows, Figure 9b, c), but not always. Some appear to grow deep into the ONL, often along Müller cell processes, where they terminate 'blindly.' This response already is prominent at 3 days of detachment. If the retina is reattached at that time and allowed to recover for a month, some of these processes persist (Figure 9c, arrows), terminating against rod synaptic endings deep in the ONL. Thus, as discussed above, not all of the rod terminals re-grow to their normal placement in the OPL. Whether those that remain in the ONL form functional synapses remains unknown.

Horizontal cells also have neurites that grow in response to detachment. These can be labeled with antibodies to calbindin D (Figure 10a; Sigma Chemical, St Louis, MO, USA), ${ }^{22}$ or neurofilament protein (Figure 10b; Biomeda, Hayword, CA, USA). A major difference between the responses of rod bipolar and horizontal cells is that neurites from the latter do not seem to seek synaptic terminals, but instead almost always grow along Müller cell processes, often following them into glial scars in the subretinal space (Figure 10b). These new horizontal cell processes may depend upon a vigorous gliotic response by Müller cells because to date we have not observed them in cases where reattachment has inhibited the growth of Müller cells beyond the outer limiting membrane.

\section{Ganglion cells respond to detachment}

We now have evidence that the third-order neurons in the retina also respond to the effects of detachment. A subpopulation of ganglion cells begins to express GAP43 , a protein mainly associated with growth of processes during neuron development (data not shown). Some ganglion cells, most likely those that also express the GAP-43 protein, also begin to show structural changes after detachment through the growth of neurites. ${ }^{23}$

\section{Non-neuronal retinal cells}

Detachment induces significant proliferation of cells that reside within the neural retina (Inset, Figure 11).
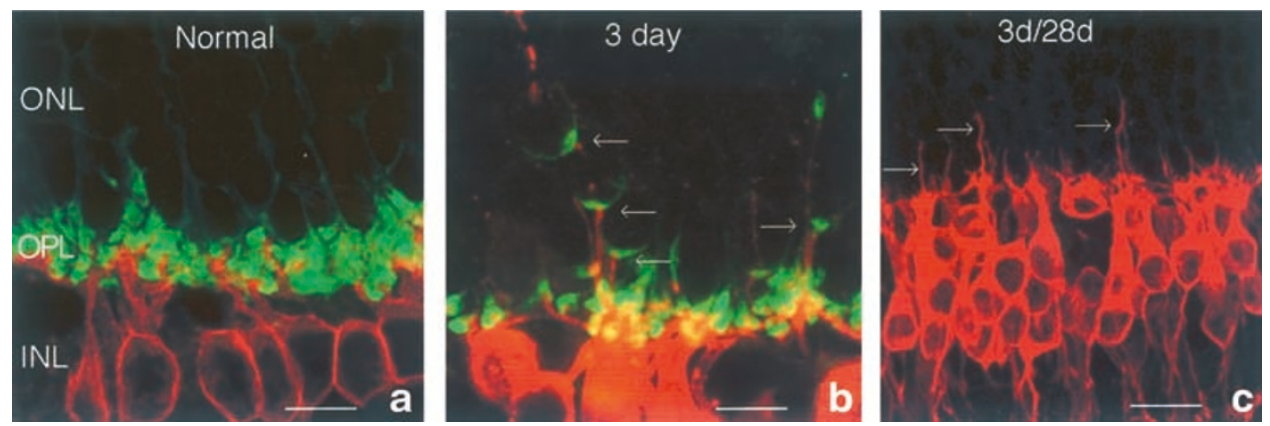

Figure 9 Confocal images of retinas immunolabeled with antibodies to protein kinase C (PKC; red), and synaptophysin (green). (a) Normal retina. Anti-PKC labels the cell bodies and dendrites of the rod bipolar cells; anti-synaptophysin labels synaptic vesicles in the terminals of rods and cones in the outer plexiform layer (OPL). (b) 3 day detached retina. Anti-PKC labels rod bipolar dendrites that extend into the outer nuclear layer (ONL) where many of these appear to contact synaptophysin labeled terminals (arrows) that have retracted toward their cell body. (c) Retina detached for 3 days and reattached for 28 days (3d/28d). Anti-PKC labeled bipolar cell dendrites (arrows) in the ONL are still present in this long-term reattached retina. (Note: this section was not labeled with anti-synaptophysin.) INL, inner nuclear layer. Scale bar, $10 \mu \mathrm{m}$ (a, b); $20 \mu \mathrm{m}$ (c). 

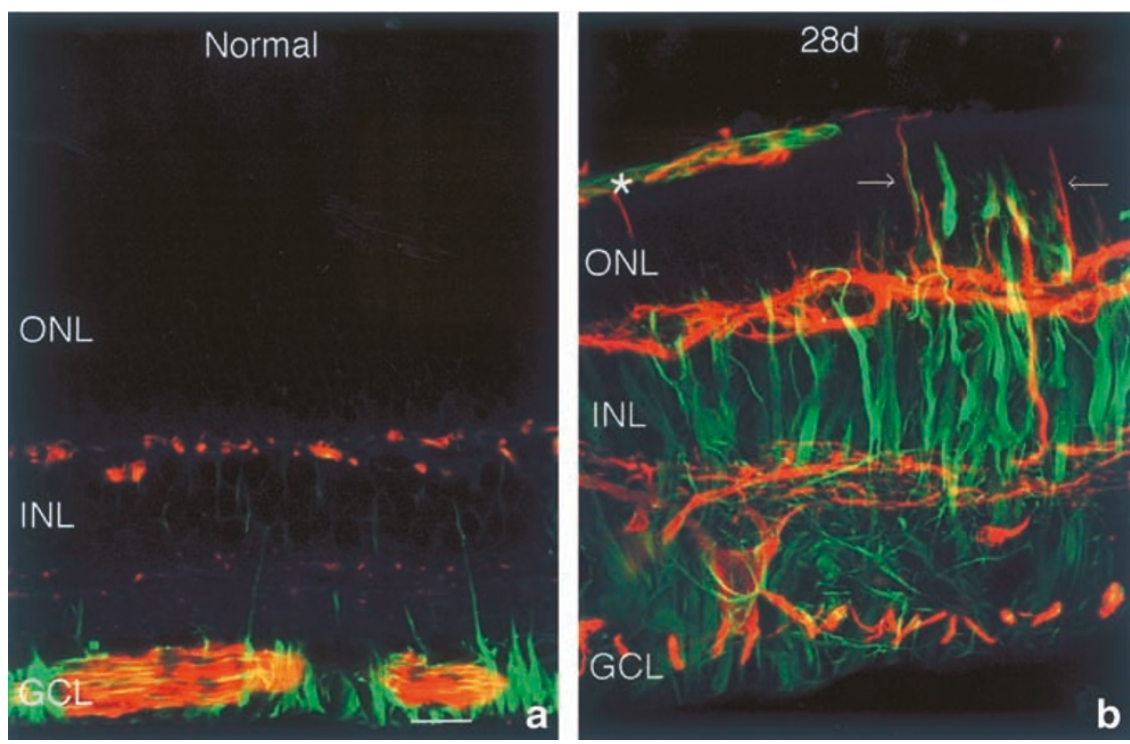

Figure 10 Confocal images of retinas immunolabeled with an antibody to neurofilament (red) and glial fibrillary acidic protein (GFAP; green). (a) Normal retina. Anti-neurofilament labels neurofilaments in ganglion cell axons in the ganglion cell layer (GCL), ganglion cell dendrites in the inner plexiform layer, and horizontal cells in the inner nuclear layer (INL); anti-GFAP labels intermediate filaments in Müller cell endfeet and astrocyte processes. (b) 28 day detached retina. Anti-neruofilament labeling is now present in horizontal cell processes extending into the outer retina, many times found growing along side GFAP-positive Müller cell process in the outer nuclear layer (arrows; ONL) and Müller cell scars $\left(^{*}\right)$ in the subretinal space. Scale bar, $20 \mu \mathrm{m}$.

\section{Cell proliferation}

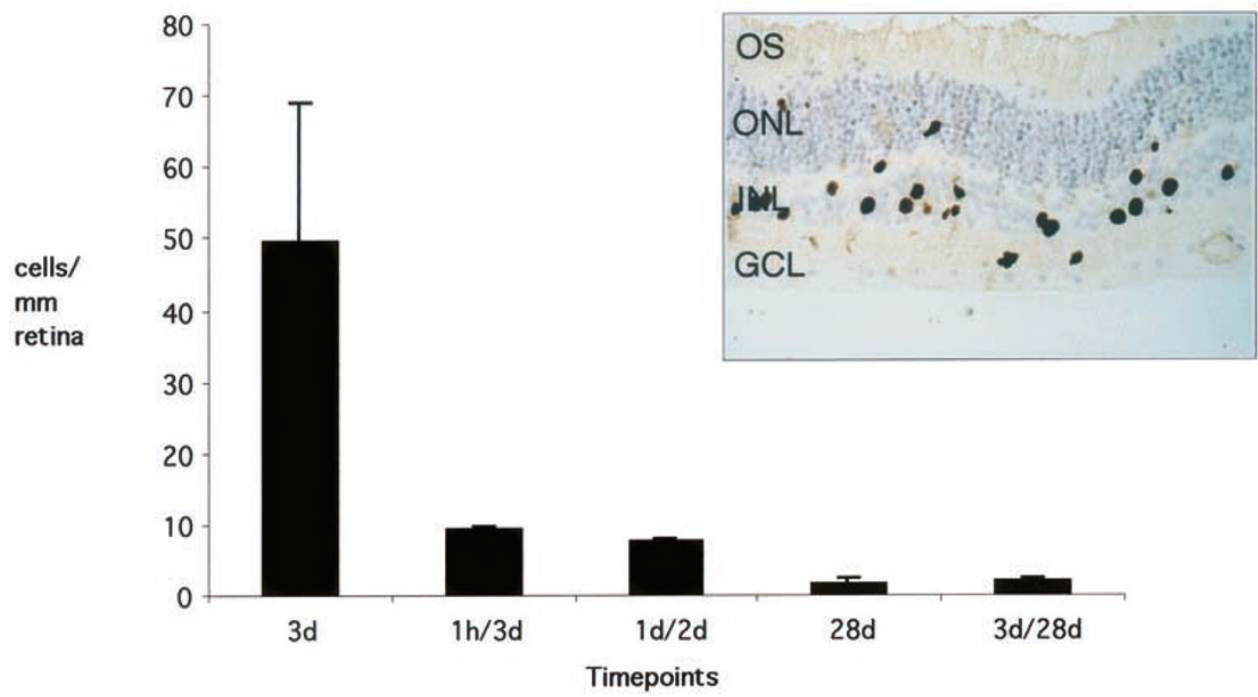

Figure 11 Graph of the number of dividing cells counted per millimeter of retina. Cell proliferation is greatly reduced in both short- and long-term detachments followed by reattachment although some proliferation is still observed in retinas reattached for 28 days (3d/28d). Inset. Example of a retina detached for 3 days labeled with the MIB-1 antibody to detect proliferating cells. OS, outer segments; ONL, outer nuclear layer; GCL, ganglion cell layer; $3 \mathrm{~d}, 3$ day detached retina; $1 \mathrm{~h} / 3 \mathrm{~d}, 1 \mathrm{~h}$ detachment followed by a 3 day reattachment; $1 \mathrm{~d} / 2 \mathrm{~d}, 1$ day detachment followed by a 2 day reattachment; $28 \mathrm{~d}$, 28 day detached retina; $3 \mathrm{~d} / 28 \mathrm{~d}, 3$ day detachment followed by a 28 day reattachment. 
Those cells in the INL labeled with an antibody that recognizes proliferating cells (MIB-1; Immunotech, Westbrook, ME, USA) can be identified as Müller cells, while smaller cells in the ONL, GCL, IPL, and sometimes the OPL, have the characteristics of astrocytes and microglia. Cells associated with the vasculature also divide although it is not clear that detachment induces significant neovascularization in the retina. ${ }^{24,25}$

Müller cells and gliosis The responses of Müller cells to detachment have been described widely. ${ }^{11}$ The earliest molecular responses to detachment have also been characterized in these cells. The extracellular signal related kinase (ERK) and the FGF-1 receptor are both phosphorylated within 15 minutes of detachment. The level of phosphorylation of the growth factor receptor drops back to pre-detachment levels by $2 \mathrm{~h}$, while the level of phosphorylated ERK remains elevated. ${ }^{26}$ Subsequently these cells become embroiled in a complex biochemical and morphological response that can jeopardize any hope for the return of vision, or provide devastating complications to retinal surgery weeks or months after the event. This response is widely known as 'gliosis,' and its hallmark elsewhere in the CNS is proliferation, growth of the glial cells (astrocytes in that case) to a greatly hypertrophied state, and a large increase in the expression of the intermediate filament proteins, glial fibrillary acidic protein (GFAP) and vimentin. These responses are particularly dramatic among Müller cells because they are quiescent in the normal retina and express very small amounts of GFAP in the unperturbed eye.

Detachment evokes a significant proliferation of Müller cells ${ }^{24,25}$ and a massive upregulation of intermediate filament proteins in their cytoplasm; GFAP (Figure 5a, b) and vimentin (Figure 6a, b; both antibodies available from DAKO, Carpinteria, CA, USA). ${ }^{27}$ These two events are accompanied by the growth of Müller cells (hypertrophy) both within the retina, where they not only fill in space left by dying photoreceptors, but grow in size and branch in the inner retina as well. In the feline model these cells seem to have a preference for growing into the subretinal space where they can form massive scars that totally inhibit outer segment regeneration (Figures $5 \mathrm{f}$ and $6 \mathrm{f})$. In fact, a single, thin strand of Müller cell cytoplasm interposed between the RPE and the reattached retina will inhibit regeneration of outer segments. ${ }^{2}$ Since such a thin strand of cytoplasm would be impossible to visualize through routine ophthalmologic observation, and would thus go unrecorded, it raises an issue of the impact of this event on visual recovery. Presumably a fine glial scar in a reattached macula could have profound effects on vision in an otherwise normal-appearing eye. Whether this actually is a significant issue in human reattachments remains unknown. The Müller cell processes can begin to grow into the subretinal space very quickly (within 3 days of a detachment). In the feline model the growing end of the Müller cell appears to have an affinity for cones or the matrix surrounding cones. ${ }^{28}$ We have occasionally observed a similar relationship between growing Müller cells and cones in samples of human retinal tissue.

Early reattachment greatly inhibits the proliferative response (Figure 11), and especially that of the Müller cells. ${ }^{29}$ Early retinal reattachment also has the ability to inhibit other aspects of the gliotic response by Müller cells, including the massive expression of intermediate filament proteins, and their growth into the subretinal space (Figures 5c, d; 6c, d). ${ }^{29}$ It appears that reattachment stops, or at least greatly slows, some of the events in Müller cells. This may not be a trivial distinction because if reattachment simply slows the response then this may permit an undesirable outcome at some later time, or it may also poise these cells to become even more reactive to other conditions of the retina such as additional injury or even aging. Does reattachment at a later time, when the response is well underway, reverse it? At present the answer is unknown. Besides being implicated in 'negative' responses to retinal injury, Müller cell reactivity may also be involved in preserving or rescuing photoreceptors. Neurotrophins can rescue photoreceptors in a variety of degenerative conditions, ${ }^{9}$ however receptors for most neurotrophins identified in retina appear to be lacking on photoreceptors, but present on Müller cells making them a prime candidate for mediating the rescue response. Likewise Müller cells may themselves secrete factors crucial to the survival of cells. It is also possible that their hypertrophy simply assures their physical presence to provide structural support for neurons and regulate the surrounding environment. Unraveling the complex responses of these cells and their role in retinal injury is in its infancy, and crucial to understanding the overall responsiveness of the retina to injury.

\section{Reattachment: creating a new environment}

Retinal reattachment may be thought of in a simplistic way of returning the retina to a 'normal' configuration. Our most recent studies of reattachment in animal models and comparisons to human reattachments indicate that this is almost certainly not true. Two events we have observed indicate that reattachment presents its own environment. 
In the detached retina we observe rod photoreceptor synaptic terminal retraction. However, only in reattachment do we observe the growth of rod terminals, with many of these growing into the inner retina (Figure 12a). We have observed similar rod axon extensions in samples of human reattachment (Figure 12b).

In the feline model of detachment we observed the growth of Müller cells on the vitreal surface with remarkable infrequency, even when the retina remained detached for weeks or months. Instead, growth was always directed towards the subretinal
CAT
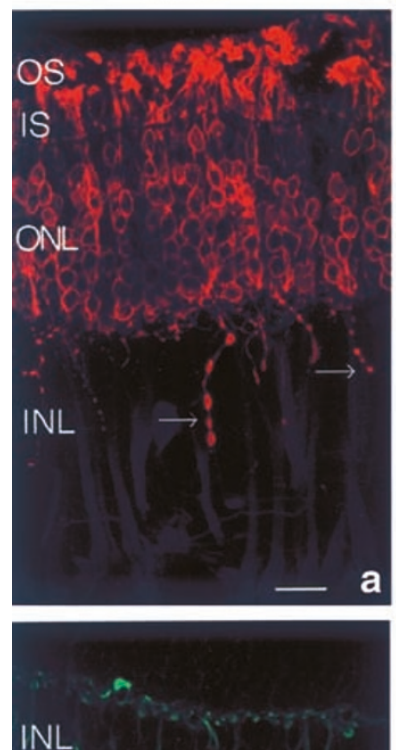

INL

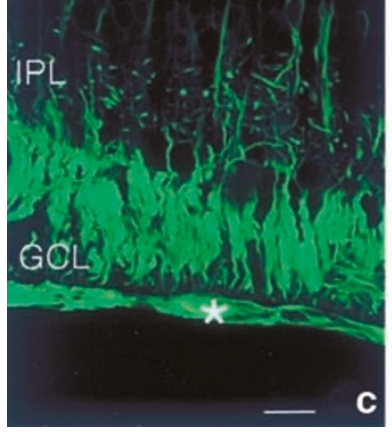

C
HUMAN
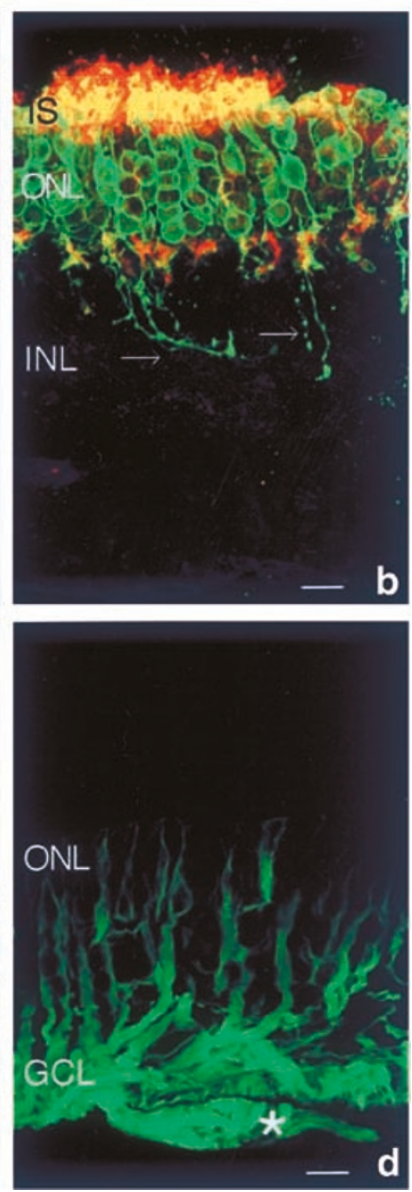

Figure 12 Confocal images of retinas immunolabeled with antibodies to rod opsin ( $a$, red; b, green) and glial fibrillary acidic protein (GFAP; c, d, green) demonstrating the similarity of the cat reattachments to human retinectomy samples taken as part of the surgery for complex retinal detachment. (a) Cat retina detached for 3 days and reattached for 28 days illustrating beaded rod axons (arrows) that extend into the inner retina. (b) Human retinectomy section showing similar rod axon extensions (arrows) to those observed in the cat reattachments. (c) Cat retina detached for 3 days and reattached for 28 days illustrating a GFAP labeled epiretinal membrane (asterisk). (d) Human retinectomy section showing a similar GFAP labeled epiretinal membrane (asterisk) to that observed in the cat. Scale bar, $10 \mu \mathrm{m}$. space. In the reattached retinas however, epiretinal membranes composed of Müller cells that grow onto the vitreal retinal surface were commonly observed (Figure 12c, asterisk). Similar epiretinal growth of Müller cells also occurs in human retinas after reattachment (Figure 12d, asterisk).

Thus, there is something special about the reattached retinal environment. It not only 'allows' the regrowth of outer segments but it also stimulates the growth of rod axons and the growth of Müller cells onto the vitreal surface.

\section{Challenges for animal research}

Retinal surgeons have developed sophisticated and remarkably effective surgical techniques for repairing retinal detachments in dauntingly challenging situations. Research on animal models probably provides the only hope for giving retinal surgeons what they don't have presently: pharmacologic agents that inhibit degeneration of photoreceptors, promote their regeneration, and inhibit the occurrence of, or cause the reversal of, devastating secondary events after successful surgical treatment. Identifying cellular targets, receptors, and ligands, that are involved in the various responses will provide a mechanism for discovering agents that can modify the response. The identification of cellular mechanisms involved in the various responses will likewise provide clues as to which molecular mechanisms need to be targeted in developing pharmaceutical strategies to treat these conditions. These identifications will almost certainly come from the use of various animal models in conjunction with the techniques of modern molecular biology.

In conclusion, outer segment regeneration may not be the major issue in visual recovery after retinal detachment. The numerous other changes that may be associated with retinal synaptic circuitry and information processing in the retina may play a significant role in this process. These include rod axon retraction and extension, changes in cone pedicles, and the growth of neurites from second-order neurons. The effect of these processes on visual recovery seems worthy of study. The proliferative response and the gliotic response of Müller cells initiated by detachment and the ability of reattachment to stimulate the growth of these cells on the vitreal surface would seem to be a major player in complications secondary to successful reattachment surgery. The role of Müller cells after detachment and reattachment may have been underestimated. Preventing their growth and hypertrophy presents a major challenge to making a significant leap in decreasing the secondary failure of reattachment surgery. 


\section{Acknowledgements}

This work was supported by grant EY-00888 from the National Eye Institute of the National Institutes of Health; Special Trustees of Moorfields Eye Hospital; The Royal College of Surgeons of Edinburgh \& The Royal Blind Asylum/Scottish National Institution for the War Blinded. CS Sethi is a Medical Research Council Clinical Training Fellow. This material were presented at the 31st Cambridge Ophthalmological Symposium, Cambridge, UK.

\section{References}

1 Steinberg RH, Reid M, Lacy PL. 1973. The distribution of rods and cones in the retina of the cat (Felis domesticus). J Comp Neurol 1973; 148: 229-248.

2 Anderson DH, Guérin CJ, Erickson PA, Stern WH, Fisher SK. Morphological recovery in the reattached retina. Invest Ophthalmol Vis Sci 1986; 27: 168-183.

3 Ohkuma, M. Transmission and scanning electron microscopic observations of the experimentally detached retina in the rabbit. I. Early changes of the detached retina. Acta Societatis Ophthalmologicae Japonicae 1972; 76: 303-315.

4 Ozaki S, Radeke MJ, Anderson DH. Rapid upregulation of fibroblast growth factor receptor 1 (flg) by rat photoreceptor cells after injury. Invest Ophthalmol Vis Sci 2000; 41: 568-579.

5 Kroll AJ, Machemer R. Experimental retinal detachment and reattachment in the rhesus monkey. Am J Ophthalmol 1969; 68: 58-77.

6 Guérin CJ, Anderson DH, Fisher SK. Retinal reattachment of the primate macula; photoreceptor recovery after short term detachment. Invest Ophthalmol Vis Sci 1989; 30: 17081725.

7 Mervin K, Valter K, Maslim J, Lewis GP, Fisher, S, Stone J. Limiting photoreceptor death and deconstruction during experimental retinal detachment: the value of oxygen supplementation. Am J Ophthalmol 1999; 128: 155164 .

8 Lewis GP, Mervin K, Valter K, Maslim J, Kappel PJ, Stone J, Fisher S. Limiting the proliferation and reactivity of retinal Müller cells during experimental retinal detachment: the value of oxygen supplementation. Am J Ophthalmol 1999; 128: 165-172.

9 Lewis GP, Linberg KA, Geller SF, Guérin CJ, Fisher SK. Effects of the neurotrophin brain-derived neurotrophic factor in an experimental model of retinal detachment. Invest Ophthalmol Vis Sci 1999; 40: 1530-1544.

10 Kroll AJ, Machemer R. Experimental retinal detachment in the owl monkey. V. Electron microscopy of the reattached retina. Am J Ophthal 1969; 67: 117-130.

11 Fisher SK, Anderson DH. Cellular effects of detachment on the neural retina and the retinal pigment epithelium. In: SJ Ryan, CP Wilkinson (eds). Retina, Vol 3. Surgical Retina. Mosby: St Louis, 2001, pp 1961-1986.

12 Guérin CJ, Lewis GP, Fisher SK, Anderson DH. Recovery of photoreceptor outer segment length and analysis of membrane assembly rates in regenerating primate photoreceptor outer segments. Invest Ophthalmol Vis Sci 1993; 34: 175-183.

13 Cook B, Lewis GP, Fisher SK, Adler R. Apoptotic photoreceptor degeneration in experimental retinal detachment. Invest Ophthalmol Vis Sci 1995; 36: 990-996.

14 Chang CJ, Lai WW, Edward DP, Tso MO. Apoptotic photoreceptor cell death after traumatic retinal detachment in humans. Arch Ophthalmol 1995; 113: 880886.

15 Erickson P, Fisher S, Anderson D, Stern W, Borgula G. Retinal detachment in the cat: the outer nuclear and outer plexiform layers. Invest Ophthalmol Vis Sci 1983; 24: 927942.

16 Wilson DJ, Green WR. Histopathologic study of the effect of retinal detachment surgery on 49 eyes obtained post mortem. Am J Ophthalmol 1987; 103: 167-179.

17 Linberg KA, Lewis GP, Shaaw C, Rex T, Fisher SK. Distribution of $\mathrm{S}$ - and $\mathrm{M}$-cones in normal and experimentally detached cat retina. J Comp Neurol 2001; 430: 343-356.

18 John SK, Smith JE, Aguirre GD, Milam AH. Loss of cone molecular markers in rhodopsin-mutant human retinas with retinitis pigmentosa. Mol Vis 2000; 6: 204-215.

19 Jacobs GH, Calderone JB, Sakai T, Lewis GP, Fisher SK. An animal model for studying cone function in retinal detachment. Documenta Ophthalmological 2002; 104: 119132.

20 Sakai T, Lewis GP, Linberg KA, Fisher SK. Hyperoxia can rescue cone-dominant retina from the effects of detachment. Invest Ophthalmol Vis Sci 2001; 42: 3264-3273.

21 Fariss RN, Molday RS, Fisher SK, Matsumoto B. Evidence from normal and degenerating photoreceptors that two outer segment integral membrane proteins have separate transport pathways. J Comp Neurol 1997; 387: 148-156.

22 Lewis GP, Linberg A, Fisher SK. Neurite outgrowth from bipolar and horizontal cells after experimental retinal detachment. Invest Ophthalmol Vis Sci 1998; 39: 424-434.

23 Coblentz FF, Lewis GP, Radeke MJ, Fisher SK. Identification of changes in ganglion cells and horizontal cells folowing retinal detachment. Invest Ophthalmol Vis Sci 2001; 42: S445.

24 Fisher SK, Erickson PA, Lewis GP, Anderson DH. Intraretinal proliferation induced by retinal detachment. Invest Ophthalmol Vis Sci 1991; 32: 1739-1748.

25 Geller SC, Lewis GP, Anderson DH, Fisher SK. The use of the MIB-1 antibody for detecting proliferating cells in the retina. Invest Ophthalmol Vis Sci 1995; 36: 737-743.

26 Geller SF, Lewis GP, Fisher SK. FGFR1, Signaling, and AP-1 expression after retinal detachment: reactive Müller and RPE cells. Invest Ophthalmol Vis Sci 2001; 42: 13631369.

27 Lewis GP, Erickson PA, Guérin CJ, Anderson DH, Fisher SK. Changes in the expression of specific Müller cell proteins during long-term retinal detachment. Exp Eye Res 1989; 49: 93-111.

28 Lewis GP, Fisher SK. Müller cell outgrowth after retinal detachment: association with cone photoreceptors. Invest Ophthalmol Vis Sci 2000; 41: 1542-1545.

29 Lewis GP, Charteris DG, Sethi CS, Leitner WP, Linberg KA, Fisher SK. The ability of rapid retinal reattachment to stop or reverse the cellular and molecular events initiated by detachment. Invest Ophthalmol Vis Sci 2002; 43: (in press). 